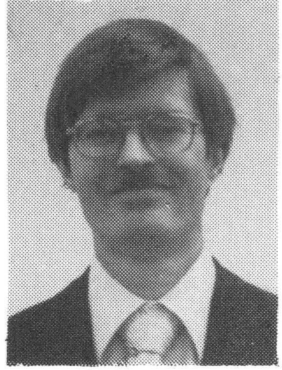

Editorial

H. J. Caulfield, Editor

On Rejected Papers

For reasons unknown to me, the number of manuscripts we must reject outright is increasing. I want to offer some advice to authors whose papers have been rejected.

First, deal with the emotions honestly and quickly. You were not rejected. Your manuscript was. You are not dishonored. The editor still welcomes future manuscripts from you.

Second, read the reviews as open-mindedly as possible. The reviewers had reasons for recommending rejection. If those reasons are valid, they saved you from public embarrassment. You should learn something and thank our good reviewers. However, the reviewers may have misinterpreted something. If they did, rewrite the manuscript to preclude that misunderstanding, write to me with your assessment, and I will ask new referees (armed with comments from the original referees) to look at your revision. Finally, the reviewers may be as wrong as they think you are. This is rare but not unheard of. This rare case of reviewer error needs special attention. You have two options for rescuing your manuscript: resubmit it to me for review or submit it to another journal. Those two options are far less different than you may think. All journal editors go to the top people in their fields as reviewers. A different journal may well use the same reviewers. It happens quite often. Furthermore, a reviewer may be annoyed if you have not revised your manuscript in response to his comments.

The object of submitting a paper is not just to publish but to communicate something true and important. Getting away with publishing a bad paper does not accomplish this purpose. The ultimate example of this approach was an author who submitted his manuscript simultaneously to Optical Engineering and to Applied Optics! Publishing is not a game. The editor takes it seriously, so you should at least pretend to. The above procedure for rereviewing a paper is complicated, but, I think, fair. It will keep a single "enemy" from killing your work. It will get your work a fair hearing. If, in the end, all of these procedures fail, I send a final rejection note.

Third, contrary to popular belief, editors do not brag about reject rates. We all hate to reject papers and hope we will, hereafter, receive only acceptable ones. We know, too, that even editors make mistakes. I hope that if we reject your revolutionary work you will have the persistence to show that we were wrong.

\section{Announcement and Call for Papers}

\section{Future Special Issue: Pattern Recognition}

Part of the November/December 1984 issue of Optical Engineering will be devoted to the topic of Pattern Recognition in honor of the 20th anniversary of the invention of the holographic matched filter by A. Vander Lugt in 1964. Authors are invited to submit research papers on this topic for inclusion in this special issue. Interested contributors should contact the Guest Editor, Joseph L. Horner, Rome Air Development Center/ESO, Hanscom Air Force Base, MA 01731 (Phone $617 / 861-5563)$.

\section{OPTICAL ENGINEERING} EDITORIAL SCHEDULE

\section{March/April 1984}

Image Scanning \& Recording Methods

Philip S. Considine

EIKONIX Corp.

23 Crosby Drive

Bedford, MA 01730

$617 / 275-5070$

Robert A. Gonsalves

EIKONIX Corp. \&

Northeastern University

360 Huntington Ave.

Boston, MA 02115

617/437-2165

Critical Technology: Infrared Optics

Irving J. Spiro

M1/129

The Aerospace Corporation

P.O. Box 92957

Los Angeles, CA $90009 \quad$ 213/615-4441

\section{May/June 1984}

Liquid Crystal Applications

Sun Lu

Crystal Vision, Inc.

1313 Geneva Ave.

Sunnyvale, CA $94089 \quad$ 408/745-0833 Ext. 221

Optical Engineering Technologies

Jerrold Zimmerman

MS 241

Honeywell Electro-Optics Operations

2 Forbes Road

Lexington, MA $02173 \quad 617 / 863-3548$

\section{July/August 1984}

Holographic Interferometry-25th Anniversary

Ryszard J. Pryputniewicz

Worcester Polytechnic Institute

Department of Mechanical Engineering

Worcester, MA 01609 617/793-5536

Precision Surface Metrology

James C. Wyant

Optical Sciences Center

University of Arizona

Tucson, AZ $85721 \quad$ 602/621-2448

\section{September/October 1984}

\section{Particle Sizing and Spray Analysis}

Gerald W. Stewart Aerodyne Research, Inc. Carnegie-Mellon Univ.

45 Manning Road

Billerica, MA 01821

617/663-9500

Dept. of Mechanical Engineering

Pittsburgh, PA 15213

412/578-2498

\section{Robot Vision}

David P. Casasent

Carnegie-Mellon University

Department of Electrical \& Computer Engineering

Pittsburgh, PA 15213 412/578-2464

\section{November/December 1984}

\section{Laser Spectroscopy}

Stanley M. Klainer

ST\&E Technical Services, Inc.

20 Belinda Court

San Ramon, CA 94583 415/829-7847

\section{Pattern Recognition}

Joseph L. Horner

Rome Air Development Center/ESO

Hanscom Air Force Base, MA 01731

$617 / 861-5563$ 


\section{Conference Report}

\section{ \\ OPTICAL INFORMATION PROCESSING CONFERENCE II}

\author{
Antonio R. Dias \\ Environmental Research Institute \\ of Michigan \\ Radar Division \\ P.O. Box 8618 \\ Ann Arbor, Michigan 48107
}

The Optical Information Processing Conference II, organized by NASA/Langley Research Center and held August 30-31, 1983, in Hampton, Virginia, convened 85 participants representing government agencies and laboratories, universities, and industry. This meeting (the second organized by NASA/Langley) brought together workers in the field of optical information processing with potential applications in NASA and Air Force programs.

The keynote speaker, Dr. John Neff [Defense Advanced Research Projects Agency (DARPA)], reviewed the field of optical processing with particular references to signal and image processing, optical computing, and hybrid systems. The need to establish a transition from one-dimensional to two-dimensional processing, which requires the development of new architectures, devices, and materials, was emphasized. New ambitious research plans, such as DARPA's Supercomputer program, will certainly foster innovative ideas.

The conference was organized into five technical sessions. The first session covered topics on Optical Data Processing Concepts. An image processing method for computer vision presented by F. O. Huck (NASA/Langley) utilizes a human vision preprocessing method. S. H. Lee (U.C. San Diego) discussed optical pattern recognition methods and showed results of software and hardware studies. A paper by C. P. Neuman (Carnegie-Mellon University) reviewed linear algebra operations realizable by optical systolic array processors. White-light optical signal processors capable of performing image subtraction, image deblurring, speech analysis, pattern recognition, and pseudocolor encoding were discussed by $F$. T. S. Yu (Pennsylvania State University), and a paper by J. B. Breckinridge et al. [Jet Propulsion Laboratory (JPL)] discussed the use of shear interferometers in white-light processing.

Optical Components and Materials Technology was discussed in the second session. L. J. Hornbeck (Texas Instruments) described a hybrid silicon membrane spatial light modulator (SLM) electrically addressable with $128 \times 128$ elements, capable of TV line rates, a peak contrast ratio of $30: 1$, and a defect level of $0.4 \%$. The Hughes silicon liquid crystal light valve (LCLV) and its applications were discussed by $\mathrm{U}$. Efron (Hughes Research Laboratory). Magneto-optical SLMs implemented with magnetic stripe domain devices were presented by J. A. Krawczack (Sperry). New exciting results in optical bistable devices (GaAsAlGaAs, fast switching, room temperature, low power devices) were discussed by N. Peyghambarian (University of Arizona). C. Warde (Massachusetts Institute of Technology) analyzed the nonlinear processing characteristics of optically addressed microchannel SLMs and electron beam addressed SLMs. Finally, a mathematical analysis of the multimode structure of channel waveguides in anisotropic crystals was presented by J. C. Bradley (Westinghouse).

The second day opened with a session on Optical Data Processing Circuits. C. S. Tsai (U. C. Irvine) reported on four laboratory hybrid integrated optical modules utilizing either acoustooptic or electro-optic effects in planar or channel waveguides. An acousto-optic device capable of obtaining, simultaneously, time integration correlation and power spectrum analysis, using commercially available $\mathrm{TeO}_{2}$ shear-wave modulators and photodiode detector arrays, was described by J. M. Pellegrino (Harry Diamond Laboratory). A single Hughes liquid crystal light valve in a flipflop configuration was suggested by S. A. Collins (Ohio State University) to implement an optical pulse generator. J. H. Goebel (NASA/Ames) discussed the usage of large format liquid crystal masks for binary optical computation.

The fourth session on Optical Data Processing Functions and Processors-l was introduced by $T$ J. Bicknell (JPL) in a talk reviewing the Jet Propulsion Laboratory's capability for ground-based processing of synthetic aperture radar (SAR) data. A. R. Tanguay, Jr. (University of Southern California) and D. Psaltis (California Institute of Technology) described designs for real-time optical processing of stripmap SAR data. Tanguay suggested the use of SLMs (CCD-LCLV, CCDdeformable mirror device, and others) as the front-end device of a classical tilted-plane coherent processor. Psaltis proposed a SAR stripmap processor implemented by using an acousto-optic (AO) cell as the input device and a CCD camera as the detector element. Both designs promise a possible range swath of 500 resolvable elements corresponding to a slant-plane resolution of about $25 \mathrm{~m}$. Unfortunately, this performance is modest compared with real-life requirements already achievable with today's compact digital implementations. P. N. Tamura (Honeywell) discussed an implementation of a broadband ambiguity function generator by optical time integration, using a surface acoustic wave (SAW) device and capable of large throughput. This session concluded with noise and system considerations on acousto-optic spectrometers used as IF spectrometers by G. Chin (NASA/Goddard).

The final session was entitled Optical Data Processing Functions and Processors-II. S. F. Habiby (Ohio State University) discussed the usage of a Hughes LCLV for optical residue addition and storage. Three presentations of matrix architectures followed. R. Athale [Naval Research Laboratory (NRL)] reviewed the outer product optical digital multiplication and an AO implementation capable of 11 bits of accuracy. A matrix partition approach was suggested by $\mathrm{H}$. J. Caulfield
(Innovative Optics) in his talk on solving large matrix problems with intermediate size optical processors. C. Verber (Battelle) discussed an integrated optics implementation of the systolic engagement architecture. A. D. Fisher (NRL) described the usage of magnetostatic waves in integrated optical signal processing, emphasizing the potential for large time-bandwidth products and simple transducers. The final paper describing a new, four-channel, two-dimensional signal processor using a single SAW delay line was discussed by C. Garvin (Harry Diamond Laboratory).

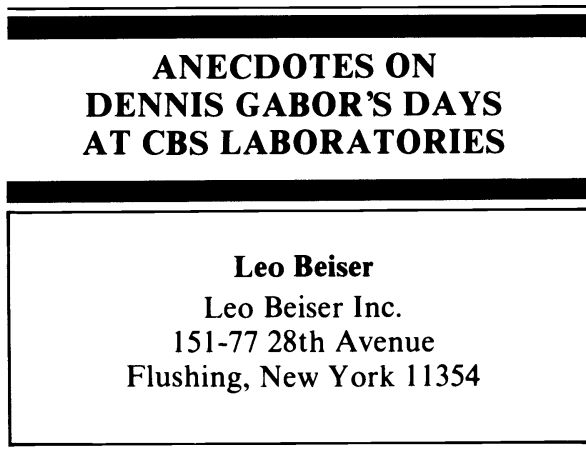

The following address was given at the Dennis Gabor Retrospective on April 20,1983, during SPIE's International Technical Conference/ Europe, which was held in Geneva, Switzerland. The program honoring the inventor of holography included a retrospective of Gabor's life and work and featured the first presentation of SPIE's Dennis Gabor Award. The texts of addresses by T. E. Allibone and E. N. Leith appear in Optical Engineering 22(4), July/August 1983, beginning on page $S R-126$.

Following Prof. Tiziani's fine introductions, Prof. Allibone's thoughtful biographical memoirs, and Prof. Leith's elegant technical review, and anticipating the appearance of Prof. Andre Gabor, who provided me with continuity of communications with Dennis during his illness, I now approach you to interweave some of the joys of Dennis' human encounters, drawing from personal and borrowed anecdotes which developed during my 13 years' exposure to Dennis' technology and humanology while at CBS Laboratories in Stamford, Connecticut.

\section{ALWAYS A TEACHER}

During a trip to Washington, D.C., with a colleague, their meetings were completed early, leaving some time available during a pleasant afternoon. Since Dennis had never seen the Smithsonian Institution, this provided an excellent opportunity to visit this world-famous museum of science and technology.

Upon entering, they observed that no tour guide was available. So, they started through the museum on their own, stopping at the great pendulum in the lobby and continuing through such varied exhibits as fire engines, clocks, steam engines, computers, power stations, and printing

Continued on Page SR-010 


\section{Throughout the World of Quality Optics}
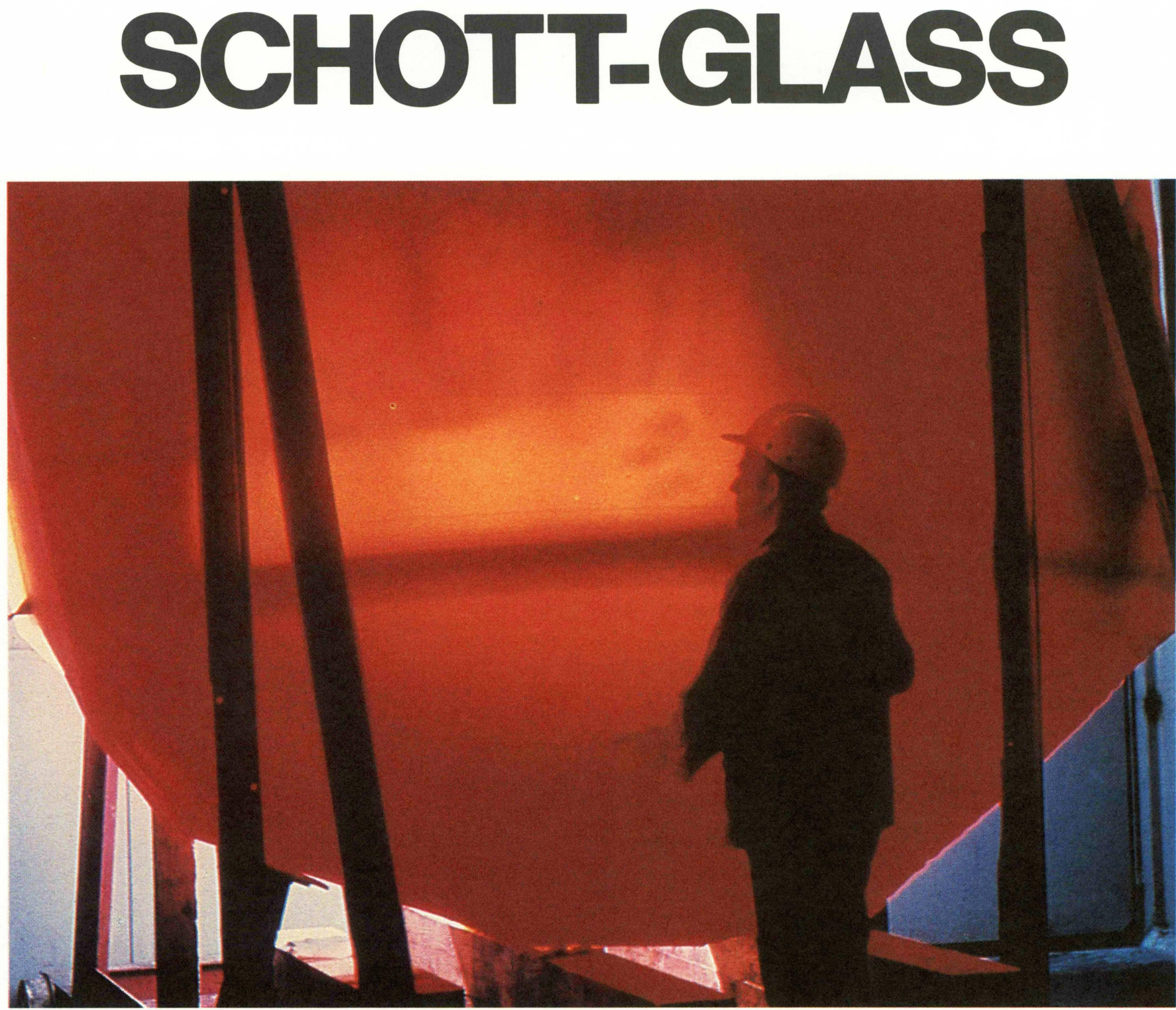

\section{Probing the infinite.}

Since ancient times, man has looked to the heavens in awe. Today, Schott technology is helping man to see farther than the ancients' dreams.

Schott manufactures Zerodur ${ }^{\circledR}$, a transparent glass ceramic especially suited for the critical specifications of reflective optics. With high Young's Modulus, practically zero thermal expansion and excellent polishing qualities, Zerodur ${ }^{\circledR}$ mirror material ensures unequalled internal quality for more uniform strain distribution.

Zerodur ${ }^{\circledR}$ has many other applications, as in laser technology, ring laser gyroscopes and in precision equipment such as modern photo-type setting machines.

Schott Zerodur ${ }^{\circledR}$ is available in discs, strips and rods in many sizes.

Contact us for your specific needs.

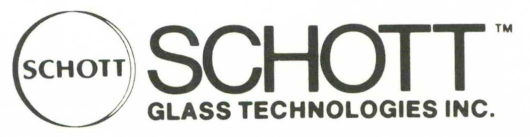

400 York Avenue

Duryea, Pennsylvania 18642

(717) $457-7485$

TWX: 510-671-4535 


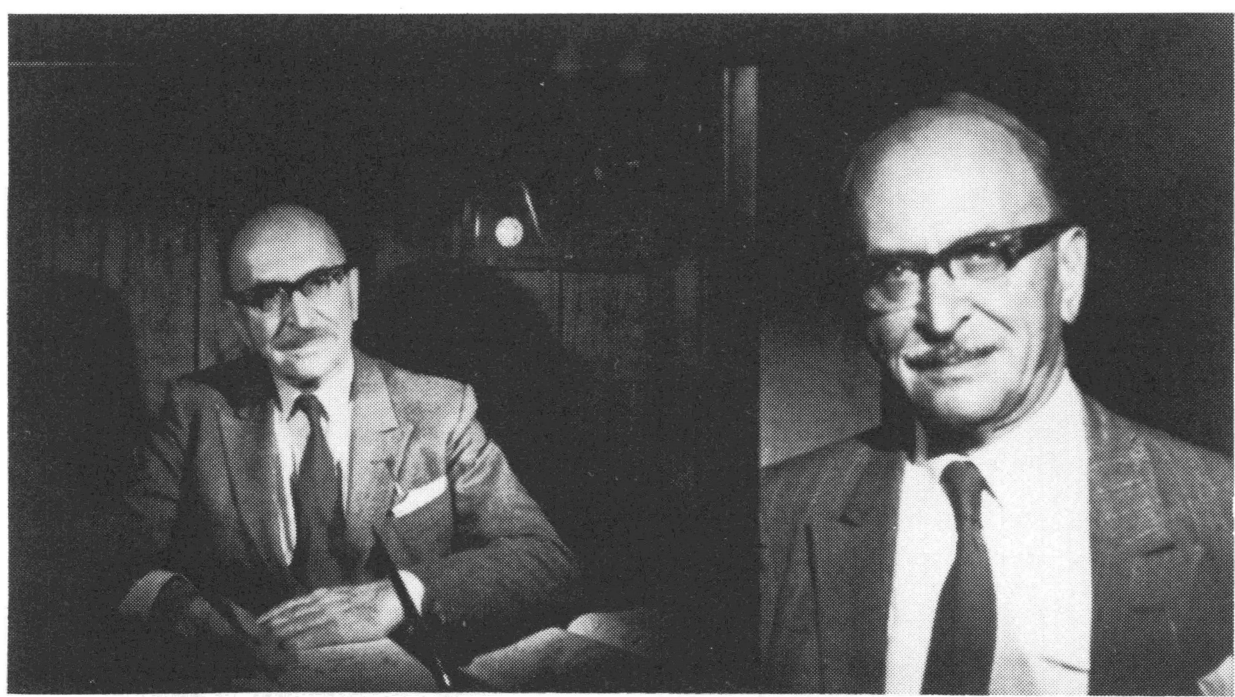

Photograph of Dennis Gabor (right) standing next to his hologram reconstruction.

\section{Continued from Page SR-008}

presses - and many more, including optics. At each exhibit, Dennis expressed to his colleague some related teachings with such clarity and perception that, unobserved by Dennis, a few passersby stopped behind him to listen. As they progressed, more Gabor tutorials of scientific wonderment continued, and more passersby accumulated, until a crowd of visitors could be seen trailing behind Dennis Gabor from exhibit to exhibit!

Thus, contrary to their first observation, there was available, at that time, a most remarkable tour guide for the Smithsonian Institution.

\section{ENERGY CONSERVATION}

Dennis' radiant energy was well known. But, after lunch and weather permitting, he would sometimes sit on a ledge outside CBS Labs' entranceway, raise his face to the warming sunshine, close his eyes and nap - a wonderful facility for recharging his batteries with solar energy.

During his so-called "retirement," when he might continue working at the office or lab at any hour of the day or night, he could switch on his energy regenerator simply by closing his eyes and dozing at his desk. "senior citizen" colleague who had also demonstrated an aptitude to "doze-off" while sitting at his desk. Well, as you might now expect, it was not unusual to find both of them facing each other, as in joint contemplation, both asleep, recharging batteries.
For a while he shared an office with another

\section{A CASE OF MISTAKEN IDENTITY}

My favorite story revolves around the portrait hologram of Dennis Gabor-one of the largest and most realistic created at that time, some $45 \times 60 \mathrm{~cm}$ (a newspaper sheet size) - which is now at the Museum of Holography in New York. When reconstructed, it provides a lifelike view, facing Dennis Gabor writing at his desk, a pen holder and an open book on his desk in the foreground, a certificate on the wood panel wall in the background.

In planning the layout of the offices and labs which were to become the Dennis Gabor Laboratories, I arranged for this hologram to be positioned near the entranceway, left side, mounted in a window-type frame in a wall. When reilluminated with the green line of an argon-ion laser, it appears as though one is looking through an open window at Dennis at his desk in his office.

One day, one of our department managers who had not yet seen this hologram was to confer with me in my new Dennis Gabor Labs. Upon entering the small lobby, he passed the hologram on his left, saw Dennis "at work," and continued to my office on his right. After completing our meeting, he left my office, and as he recrossed Dennis' image on his right, he glanced down over his shoulder at Gabor at his desk and said quietly, "Hi, Dennis." With a wave of his hand he continued, closing the main door softly behind him.Let me remind you that Dennis, and everything else in his "office," was $5145 \AA$ green!-I counted the seconds for our colleague to walk toward his office, come to a screeching halt, dash back, thrust open the door to my Labs, and proclaim, "But Dennis is not here today!"

No, Dennis is not here today, but his cherished image lives forever.

\section{Book Reviews}

\section{Electron and Ion Microscopy and Microanalysis: Principles and Applications}

Lawrence E. Murr, (Optical EngineeringVolume 1, Brian J. Thompson, Series Editor), xiv + 794 pp., illus., indexes, references, appendixes. ISBN 0-8247-1553-5. Marcel Dekker, Inc., 270 Madison Ave., New York, NY 10016 (1982) \$65.

Reviewed by (Part I) Thomas A. Emma, GTE Laboratories, 40 Sylvan Rd., Waltham, MA 02254, and (Part II) N. Carlile Miller, Equipment Development Labs., Raytheon Co., Sudbury, MA 01776.

Part I. Murr's book is an extension of his earlier work Electron Optical Applications in Materials Science. The text covers an extensive range of material and provides the interested reader with a comprehensive collection of applications and reference material. It should therefore provide excellent material for a course in electron microscopy. The book is perhaps inappropriately titled since it deals with electron beam instruments and applica- tions and only introduces ion optics. The major strength of the book is its many applications to materials science as well as the selection of problems. Many of the applications are taken from Murr's own work, and many are classical examples of applications taken from other sources. The development and explanations of each application are carefully considered and presented in a lucid manner. The selection and presentation of line drawings is excellent. The subjects considered in most detail are those covered in Murr's earlier work; the extension of these to include recent developments is, in most cases, excellent.

The presentation of fundamental concepts in the early chapters is, however, slightly uneven; some areas are covered in extensive detail while others of equal importance are not treated proportionately. For example, the development of the motion of electrons in a periodic potential using the Kronig-Penney model and the Bloch scheme for generating dispersion relations is not extended later on when the dynamical theory is introduced. In some of these areas the use of unconventional notation and the presence of typographical errors may be misleading for students attempting a self- study course. For example, Brillouin zones are not forbidden zones; their boundaries define the forbidden levels.

Although Murr introduces and treats the subject of electron diffraction extremely well, his treatment of convergent beam methods is too limited for a modern text. This important subject is included in the areas the text treats well and deserves more attention.

In general, the text is a successfully expanded and updated version of the author's original book and provides many useful examples of applications and interpretation, as well as an excellent development of specimen preparation techniques. The inclusion of reference charts and tables should be most helpful to any student of the subject.

Part II. The comments of this reviewer are directed mainly at the chapters devoted to electron probe microanalysis and scanning electron microscopy.

In contrast to the rest of the book, the treatment of electron and ion probe microanalysis in Chap. 4 contains many deficiencies. The major topics are covered in enough detail, at an appropriate level for an introduction, but there are a 
surprising number of omissions and inaccurate statements. In particular, the discussion of quantitative electron probe microanalysis in bulk, flat materials should include the atomic number effect; should discuss $\mathrm{x}$-ray spatial resolution; should outline or refer to specific correction models; and should mention the subject of accuracy. The advantages and disadvantages of wavelength and energy dispersive spectrometry should be described.

In addition, resolution and sensitivity are confused in Sec. 4.4. The section on Auger electron spectroscopy does not describe the Auger process correctly. Spatial resolution of one micrometer is claimed for ESCA. The microanalysis summary table (Table 4.3) is very helpful, but does contain a number of errors.

Some terminology and some of the section titles are not consistent with common usage. The material on sample preparation for electron probe microanalysis is too brief. However, in Appendix B many valuable techniques for replica and thin foil preparation are described. A number of very interesting applications of electron probe microanalysis can be found in Chap. 4. The subject of microanalysis of thin foils is treated well.

In Chap. 5 the author presents very well the concepts of scanning electron microscopy, such as secondary electron spatial resolution and image formation. All major types of information obtained in the SEM are described. A number of excellent applications are given. A comprehensive list of references is also included.

\section{Acousto-Optic Signal Processing (Theory and Implementation)}

Norman J. Berg and John N. Lee, eds., (Optical Engineering-Volume 2, Brian J. Thompson, Series Editor), 504 pp., illus., index, references with each chap., bibliographies at end. ISBN 0-82471667-1. Marcel Dekker, Inc., 270 Madison Ave., New York, NY 10016 (1983) \$65.

Reviewed by Milton Gottlieb, Westinghouse R\&D Center, Pittsburgh, PA 15235.

Optical processing of real-time signals using acoustooptic techniques is currently in the process of incorporating new, significant advances into systems that look less and less like laboratory demonstrations and more and more like fieldable hardware. This has generated considerable enthusiasm in some circles for the potential of this technology; it is not, however, the first time such enthusiasm has been generated. There have been at least two periods of intense activity since the early 1960s, followed by some disillusion as expectations could not be satisfied. Hopefully, the current enthusiasm is more firmly rooted, not only in a better understanding of what is possible, but also in a variety of recent technologies that permit the design of systems with performance that was not realizable a decade ago. The book covered in this review describes some of the advances that have made this possible, and gives a realistic assessment of the opportunities, as well as the limitations, of acousto-optic signal processing.

The book is divided into four main parts: these are an introduction to acousto-optics, frequencydomain signal processing, time-domain signal processing, and integrated optics. Each of these parts contains anywhere from one to five chapters, each chapter having been contributed by an expert (or group of experts) on that subject. Since an expert will usually have strong opinions on the proper way to approach a project (different from other experts), it is not surprising that these chapters differ widely in the way they are written. Fortunately, each tends to be quite self-contained, with a generous helping of tutorial material, so that many readers may find it appealing to read only those chapters that may be of immediate interest, and in any order. Others might find it most useful to read the chapters in sequence to gain overall familiarity with the entire field.

The first part of the book contains three chapters, and is an introduction to the basic acoustooptic phenomenology and Bragg cell design considerations. It is possible to present the mathematical treatment of acousto-optic interactions with many levels of complexity, depending upon the purpose of the presentation. A relatively simple, nonmathematical treatment will, in my opinion, suffice for a working understanding of signal processing devices. The theory in the introduction of this book exceeds this requirement, and is in some ways too exotic in approach for the intended audience. It will be discouraging to many readers who choose to follow each chapter in sequence. The chapter on Bragg cell design was written by a group of contributors from Harris Corporation, and represents their approach to the optimization of modulators and deflectors. A brief section on materials and transducer fabrication is included, with good references to the more comprehensive literature.

The heart of the book is contained in parts II and III, which deal directly with signal processing. Part II is devoted to frequency domain signal processing and consists of three chapters. RF spectrum analysis has been identified as perhaps the single most important application of acoustooptic processing, and J. P. Lindley has written a very concise and readable chapter on this application. It is entirely nonmathematical and attempts only a broad description of the highlights and state of the art of acousto-optic spectrum analyzers. The reader with a serious interest in this application will most certainly want to read further in the available literature. The following chapter, Coherent Detection and Adaptive Filtering by D. W. Jackson and J. L. Erickson, deals with a related application, the optical excisor, whose function it is to remove selected frequencies from a broadband spectrum, in a real-time, adaptive fashion. An analysis of excision is carried out, in which conventional filter theory is applied to the optical excisor. This is followed by a sizable section on computer-generated filter response for a range of parameters. Some interesting experimental results are shown, but it would have been helpful if more details of the optical system could have been included. One of the interesting experiments simulated a self-adaptive notch filter made by optically writing the blocking regions using the PROM. Although this will not be practical because of cycling problems, it is a convincing demonstration of the power of optical processing. The third chapter in this part is Acousto-Optic Tunable Filters by I. C. Chang, who is a leading authority on this subject. The acousto-optic tunable filter uses RF signals to analyze optical energy (like a spectrometer) and so is totally different in character from all of the other signal processing devices that are the subject of this book. This chapter is a brief, easily understood summary of the principles of operation and performance characteristics of various types of AO tunable filters, and several applications. There is a great deal more to this important area of acousto-optic devices, but this book would not be the appropriate place for a more lengthy exposition.

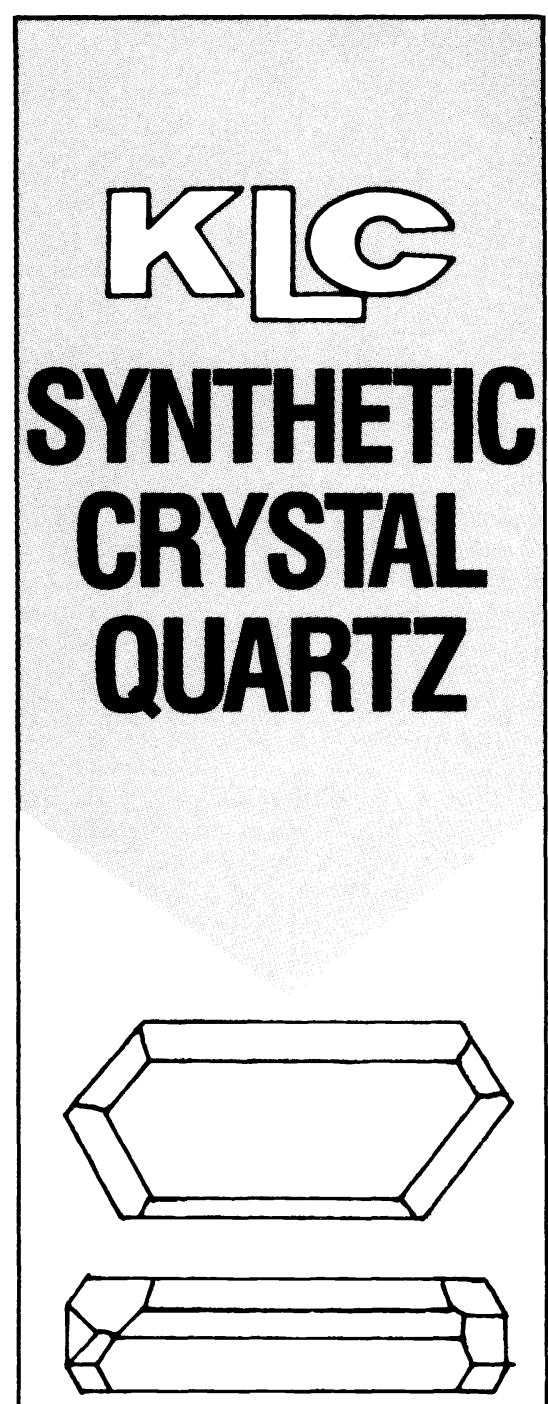

KARL LAMBRECHT CORP. foremost supplier of Polarizing Optics for fifty years, now offers Synthetic Crystal Quartz in AsGrown Crystals as shown above, Blanks to your specs, or Finished Optics CALL OR WRITE FOR DETAILS

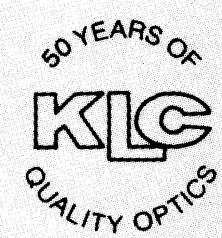

KARL LAMBRECHT CORPORATION $4204 \mathrm{~N}$. Lincoln Ave. Chicago, II. 60618 USA Phone (312) $472-5442$ TELEX: 254771 LAMBRECHT CGO. 
The third part of the book, time-domain signal processing, deals with those powerful applications of acousto-optic devices that utilize the ability to spatially modulate light over an extended time interval, leading to the fundamental operations of convolution and correlation. Here also are descriptions of many of the new processing architectures embodied in acousto-optic systems, such as time-integrating correlation and triple product processing. It is very largely due to the realization of these powerful algorithms that interest has been renewed in $\mathrm{AO}$ processing. There are five chapters in this part, the first being an excellent review by Casseday, Berg, and Abramovitz entitled SpaceIntegrating Acousto-Optic Signal Processors Using Surface Acoustic Wave Delay Lines. Although the particular experimental system about which all of their work revolves is the lithium niobate SAW delay line in which a sheetlike beam of light is externally focused near the surface on which the acoustic waves propagate, the algorithms they describe can be realized as well with bulk AO systems and integrated optic systems. These include the basic time- and spaceintegrating correlators, the chirp- $Z$ transform implementation, and a multichannel correlator. Included are the simple triple product convolver, the triple product convolver with CCD chip-Z transform modules to form very long discrete Fourier transforms, and the two-crystal real-time correlator. For all of these examples the mathematics is very clearly presented, with a minimum of complexity. It is gratifying that they illustrate most of these examples with experimental results; in some cases these experiments have been rudimentary, but they serve as a proof of principle and lend credibility to the approach. The next, brief chapter in this part, Signal Processing Using an Acousto-Optic Memory Device, by J. N. Lee, is more tentative in nature, concerning itself with real-time storage mechanisms in materials such as lithium niobate. The most important such mechanism, the acoustophotorefractive effect, results in erasable refractive index changes, replicating those present in a surface acoustic wave at the instant of exposure to a short duration, high peak power laser pulse of appropriate wavelength. Various related storage mechanisms are described, although these are not well understood. Lee summarizes the measured experimental parameters in lithium niobate as they might apply to signal processing applications, and shows some results with a memory correlator for linear FM chirp and binary coded waveforms. He makes it clear that a practical system is far from present reality, and progress in this field will be worth following. The third chapter in this part is Incoherent-Light Time Processors, by J. D. Cohen. It is entirely analytical and is a review of various types of processor architectures which use incoherent light sources such as LEDs. There are many time-integrating correlator architectures, including interferometric and noninterferometric, and the major portion of the chapter is devoted to these. I found this material to be very well organized, and the complex mathematics well laid out for easy following, with excellent diagrams of the electrical equivalents of the various optical processors. The following chapter on Coherent Time-Integration Processors, by Abramovitz, Berg, and Casseday, is also an excellent summary of a large volume of material, although quite different in style from the previous chapter. The analysis covers a variety of coherent processor architectures, including the two-beam time-integrating correlator, and one and twodimensional processor systems. Although these may be implemented with either bulk or surface acoustic wave devices, all of the experimental work illustrated utilizes the surface acoustic wave delay lines with focused sheet optical beam. Their examples include spread spectrum processing for synchronization and time difference of arrival, and these have demonstrated large processing gain with field prototype hardware. The two-dimensional system example is the range-Doppler processor, which may also be used for twodimensional spectrum analysis (coarse and fine resolution). The final chapter of this part is by $D$. Casasent, Optical Information Processing Applications. It is also primarily analytical and deals with such EW applications as ambiguity function processors, spread spectrum communications, and several multichannel systems for direction of arrival determination. Considerable emphasis is placed on the need for, and methods of, handling complex-valued data. Two new, advanced acoustooptic processor concepts are described here; these are a space variant processor, and a hybrid timespace integrating correlator. It is clear that these are very powerful concepts which will allow optical processors to address very sophisticated problems in the future.

The final part of the book consists of one chapter on Integrated Optics, written by D. Mergerian and $E$. Malarkey. If optics represents the future of signal processing, then it may be said that integrated optics represents the future of optical processing. For indeed, ths embryonic technology embodies so many of the potential advantages, such as small size, ruggedness, and batch fabrication capability, that must be attained. Mergerian and Malarkey have pioneered the development of the first integrated optic spectrum analyzer, and write with authority on this subject. The treatment consists of a review of the basic principles of light propagation in dielectric waveguides, followed by a discussion of the integrated optic components important for signal processing. They do this on a level intended for the reader who is not a specialist in optics, and therefore there is a minimum of mathematics. It is well written and illustrated, and easily understood. While many types of interactions are possible with guided light, they concentrate on those with surface acoustic waves for signal processing. There is an excellent summary of the design and fabrication principles of waveguide lenses, many of which have been developed in their laboratory. Finally they describe a few of the integrated optic signal processing devices that have been developed in the past few years. This includes the RF spectrum analyzer, an A/D converter, a time-integrating correlator, and a high speed optical switch. They end with a very comprehensive bibliography of the integrated optics literature.

To summarize my evaluation of this book, I would be inclined to characterize it more as a collection of research progress reports than as a textbook on acousto-optic signal processing. There is too great a disparity of style from chapter to chapter, the sequence of material is not entirely rational, and there is considerable overlap and repetition between chapters. On the other hand, each chapter is essentially self-sufficient, and may be read individually as a review of that portion of the field. It will be a fine source book for the graduate student or for any new arrival to optical signal processing, and I can recommend it highly as a desk reference for anyone actively involved in acousto-optics.

\section{Digital Image Processing: A Systems Approach}

William B. Green, 203 pp., illus., index, references, appendixes. ISBN 0-442-28801-8. Van Nostrand Reinhold Company, Inc., 135 West 50th St., New York, NY 10020 (1982) \$34.50.

Reviewed by T. S. Huang, Coordinated Science Laboratory, University of Illinois at UrbanaChampaign, 1101 W. Springfield Ave., Urbana, IL 61801 .

In a recent IBM Europe Institute on Digital Image Processing, Professor A. R. Forrest, University of East Anglia, gave a lecture on computer image synthesis. During his lecture, he compared computer graphics with image processing and made a statement along the following line: "In computer graphics, we are very pragmatic. Usually we do not have much sophisticated mathematics. But our simple algorithms do their jobs." To which I offered this response: "Yes. And in image processing we have a lot of complicated mathematicsmost of which does not work."

Well, here is a book that talks about the working part of digital image processing. In the author's own words, "The main emphasis of the book is in the reduction of theory to practice. Most of the material deals with issues related to implementation of image processing systems."

The book contains seven chapters: (I) Introduction, (2) Digital image acquisition systems, (3) Subjective image processing techniques, (4) Quantitative image processing techniques, (5) Image display, (6) Image processing soft ware design concepts, and (7) Image data base management. Chapter 4 covers histogram modification, false color, color compositing, and high-pass filtering. Chapter 5 covers geometric and radiometric corrections, multispectral classification, and color manipulation.

In addition, there are three appendixes: (1) Data sources, (2) Available NASA computer software and documentation, and (3) Bibliography. The first two are very useful, the bibliography is rather brief.

The book contains many image processing examples (some in color), a majority of which are from the Jet Propulsion Laboratory (JPL). The author, now at System Development Corporation, spent ten years at JPL.

The book under review is quite different from other texts on digital image processing (Rosenfeld and Kak, Pratt, Hall, Gonzales and Wintz, Castleman). Since it takes a systems approach, individual topics are necessarily treated rather shallowly, and some standard topics (e.g., shape and texture analysis) not at all. In spirit it is closest to the text by Castleman (who is at JPL). Indeed, between Green and Castleman, one gets an excellent view of the digital image processing work done at JPL.

Note that except for Green and Castleman, all the other texts mentioned above were written by professors (Pratt and Wintz have since turned to greener pastures). We professors are notorious for our fondness of sophisticated mathematics. Working engineers should be forewarned that the mathematics is there mainly to give one insight; much of it may not lead to practical algorithms.

In my opinion the best audience for the book under review is the engineer just getting into the area of digital image processing. Students (or their professors) may not find the book deep enough. 


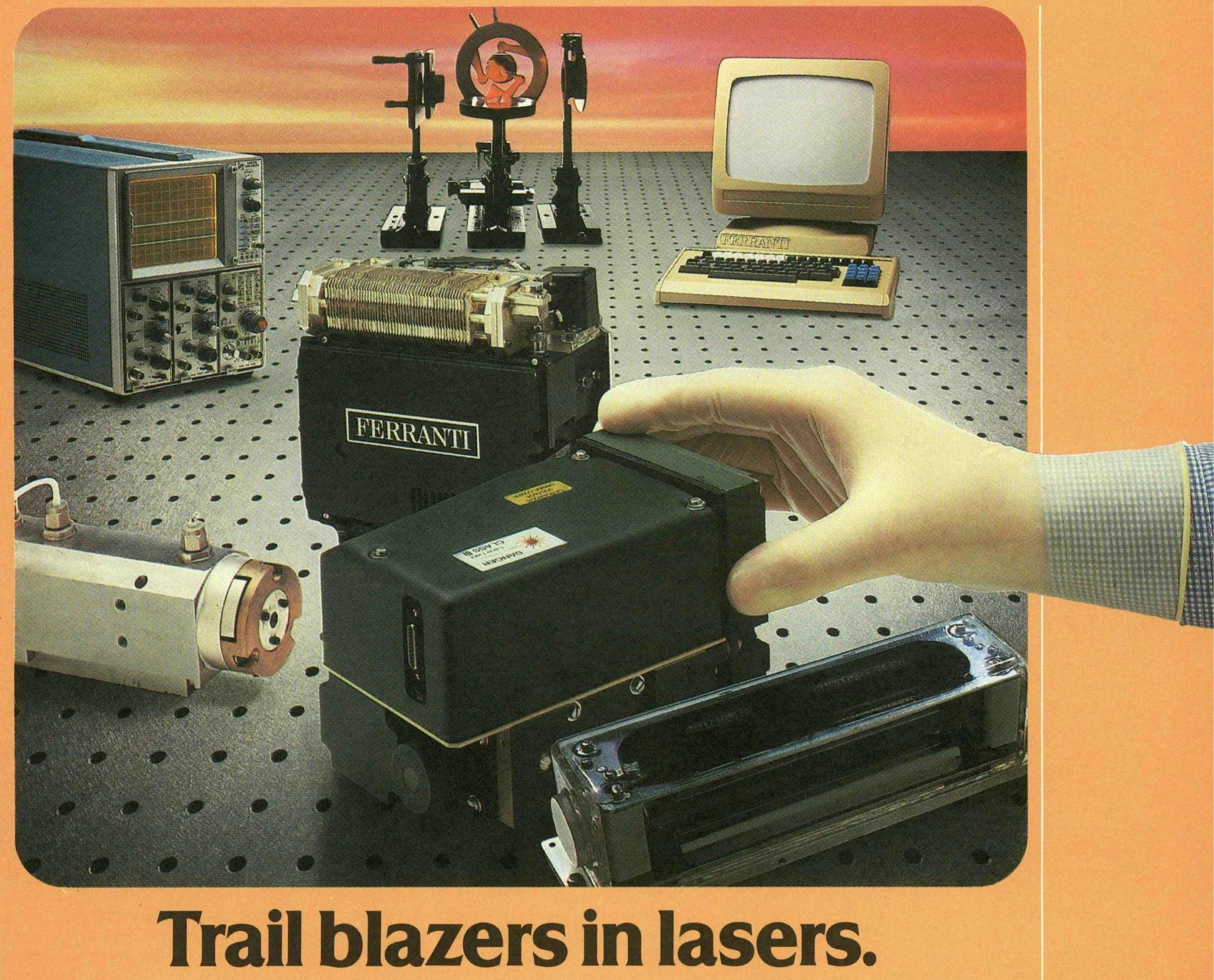

Now you can buy laser systems with the most advanced technology, direct from Ferranti in the United States. The four types we show here are not just laboratory specimens. They are ruggedised so they can be used in the field as part of a prototype system.

Ferranti can offer fast delivery from its new facility at Huntington Beach, California.

Ask about:

Nd YAG Mini Rangefinder (Type 520)

Nd YAG Rangefinder/Designator (Type 629)

$\mathrm{CO}_{2}$ Eye-safe Sealed T.E.A. Transmitter (Type 630)

$\mathrm{CO}_{2}$ 1-20W RF/DC excited waveguide laser (CM Series)

Ferranti Electro-optics Inc.

16812 Gothard Street,

Huntington Beach,

California 92647, U.S.A.

Telephone: 714.8416812

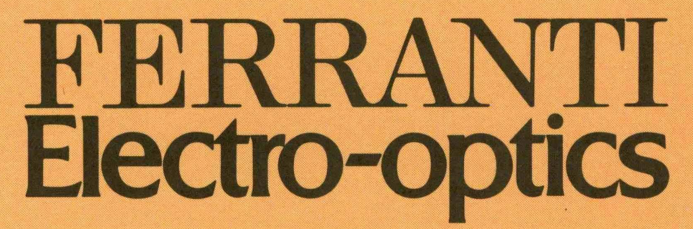


Continued from Page SR-012

\section{The Exploration of Outer Space with Cameras: A History of the NASA Unmanned Spacecraft Missions}

Michael M. Mirabito, vi + 170 pp., illus., index, references. ISBN 0-89950-061-7. McFarland \& Co., Inc., Box 611, Jefferson, NC 28640 (1983) $\$ 19.95$.

Reviewed by Farouk El-Baz, Itek Optical Systems, Lexington, MA 02173.

No one doubts that nothing has aroused sustained, world wide interest as much as planetary exploration did during the past two decades. Images transmitted by spacecraft across vast distances constituted the most tangible evidence and the easiest to comprehend among the results of the space voyages. Fascinating images of the planets and their moons swiftly reached the remotest desert settlements and mountain villages via dissemination by public media throughout the world. These sharp and clear views of the alien worlds that remained shrouded in mystery for so long have already permeated nearly every textbook on the natural and physical sciences throughout the educational system in the United States. This new treasure of solar system vistas was the outcome of well-placed emphasis by NASA managers and their advisory scientific groups on imaging systems for the planetary exploration missions.

This book is a description of these American unmanned space missions, which unveiled the planets and their moons, including the Ranger, Surveyor, and Lunar Orbiter missions to Earth's Moon; the Mariner mission to Mars and Mercury; the Viking Orbiter and Lander explorers of Mars; the Pioneer missions to Venus and the outer planets; and the Voyager series to Jupiter, Saturn, and beyond. After a survey of the various spacecraft and their imaging systems ( $82 \mathrm{pp}$.), the book includes a short, five page section entitled Camera Calibration and Image Processing; an analysis of the photographs of the various solar system bodies $(20 \mathrm{pp}$.); a summary of proposed outer space missions ( $15 \mathrm{pp})$; a chapter on terrestrial applications of spacecraft cameras (7 pp.); and a two page chapter entitled Implications and Conclusions.

The technical heart of the book includes the description of the cameras that were used to obtain the images. The various spacecraft series are described in nearly an identical manner with an introductory statement, a section on design criteria, one on camera design, another on camera optics, and a conclusion. This rigid structure allows the author to be methodical and thorough, but it gives the book the character of a dull thesis rather than a lively synthesis. It also dictates repetition of numerous statements, particularly in the many introductions and conclusions.

The reliance on prose rather than line drawings to describe intricate systems is a major failing of the book. Only a specialist can follow the descriptions, and no vivid "image" is imprinted in the end. The investment in the production of many of the 32 plates, such as the photographs of spacecraft or their models, would have been much better placed on good, clear line drawings of the imaging systems.

Another failing of the book is that it completely lacks any consideration of the human aspects of the space missions and sensu stricto should not be considered a "history." The spacecraft and their imaging systems are discussed as if they were borne of robots that were controlled by computers. In reality, these imaging systems were selected at times because of the clout of certain groups of scientists, at other times because of cer- tain developments and breakthroughs in imaging technology, and at yet other times because of power politics and budget constraints within and without NASA. All of these parameters varied from time to time, depending on perceptions as well as policies. The lack of this human dimension could have easily been remedied by a sprinkling of firsthand comments or interviews with individuals that served with NASA, its advisory groups, or its numerous contractors in the private industry. Most of these people are still living and are quite accessible.

With all its failings, the book is vastly more readable than the many NASA publications that include the same information. It also has the distinction of being the only book that groups together the imaging systems of all the American unmanned space missions to date. It is recommended to those who are interested in the planetary missions in general and their imaging systems in particular, but the reader should not expect an intellectually stimulating synthesis.

\section{Probability, Statistical Optics, and Data Testing: A Problem Solving Approach}

B. Roy Frieden, 404 pp., illus., index, references ISBN 3-540-011769-5, Springer-Verlag Publishing Co., 175 Fifth Avenue, New York, NY 10010 (1983) \$39.

Reviewed by Rodney Shaw, Eastman Kodak Company, Research Labs., Rochester, NY 14650.

The author rightly states in his preface that a basic skill in probability is currently demanded in many branches of optics, and especially in image science. Noting the lack of texts that deal with stochastic processes and statistics from the optical perspective, the author states his intent to fill this void and by and large he has done so with success, and certainly with infective enthusiasm.

If we glance at the chapter headings (The Axiomatic Approach; Continuous Random Variables; Fourier Methods in Probability; Functions of Random Variables; Bernoulli Trials and Limiting Cases; The Monte Carlo Calculation; Stochastic Processes; Introduction to Statistical Methods; Estimating the Mean, Median, Variance, $\mathrm{S} / \mathrm{N}$, and Simple Probability; Estimating a Probability Law; The Chi-Squared Test of Significance; The Student t-Test on the Mean; The F-Test on Variance; Least-Squares Curve Fitting-Regression Analysis; Principal Components Analysis; The Controversy Between Bayesians and Classicists), we see nothing to tell us that this is anything more than just another book on probability. But then we recall that the title promised us both statistical optics and a problem solving approach, and this is precisely what we get.

The author has indeed made an exhaustive effort to keep the text both practical and relevant. With a profusion of problems from imaging and optics and numerous worked examples and student exercises, the tools of probability and statistics come into focus before our eyes: computed tomography, underwater illumination, unsharp masking, atmospheric turbulence, laser speckle, film grain noise, maximum entropy restoration of galactic images, absorptance of optical fibers; these are just a few of the topics that are discussed. At this point it is appropriate to dispense with the small amount of bad news. It is scarcely surprising that amongst this array of illustrative examples we should find some superficiality of treatment, and the author tends to shoot from the hip at times. Specialists in the various imaging fields may find slightly irritating examples of this among the topics they know best.

The good news is large, however. The author has produced a text that by its practical nature and quality will prove to be a constant bookshelf companion to many of the readers of Optical Engineering. It is also of considerable satisfaction to this reviewer to know that, since no doubt the text is drawn from Professor Frieden's own lecture courses in the optical sciences, a generation of optical students is thus being introduced to such stimulating material and such an original approach.

The author has organized the flow of the text with skill, and the book is nicely produced in general; at $\$ 39$ it is a good value. The literary style is a little racy at times, but maybe that is what it takes to command student attention these days.

Take a look at this book as soon as you can. The odds are that you will find something useful and interesting.

\section{Measurement of Suspended Particles by Quasi-Elastic Light Scattering}

Barton E. Dahneke, ed., 570 pp., illus., index, references. ISBN 0-471-87289-X. John Wiley and Sons, Inc., Order Dept., 1 Wiley Drive, Somerset, NJ 08873 (1983) \$44.95.

Reviewed by Alex M. Jamieson, Dept. of Macromolecular Science, Case Western Reserve University, Cleveland, $\mathrm{OH} 44106$.

This text provides a useful and coherent review of theoretical principles and the current experimental status of quasi-elastic light scattering (QLS) techniques for the characterization of colloidal dispersions. The book is based on a symposium organized by the editor, and consists of 20 uniformly well written chapters, representing papers contributed by different authors, grouped in four sections. Section 1 comprises two excellent and authoritative introductory chapters. The first, by R. Pecora, deals with theoretical principles and a review of applications of QLS to investigate dynamical behavior of macromolecules in solution; the second, by N.C. Ford, provides primarily a discussion of experimental procedures and current instrumentation.

Section 2 consists of a series of papers describing the current status of computational methodology for inversion of experimental correlation functions to generate information on the relaxation spectrum and hence derive data regarding the particle-size distribution. These contributions provide a very complete and balanced description of alternative numerical procedures. As discussed in a clear and concise summary by E. F. Grabowski and I. D. Morrison (Chap. 7), these alternatives are (1) nonlinear least squares fit to an assumed distribution function, which is the method of choice for those systems where such information is available; (2) Laplace inversion of the correlation data to generate the smoothest non-negative distribution, an approach detailed in Chap. 5 by S. Bott and utilized successfully in several different laboratories, including our own, in the form of the program CONTIN, widely distributed by its author, $\mathrm{S}$. N. Provencher (see Chap. 5); and (3) the histogram method first applied by E. Gulari et al., also successfully used by many laboratories, and described 
in detail in papers by B. Chu and A. DiNapoli (Chap. 3) and B. Bedwell, E. Gulari, and D. Melik (Chap. 8). Chapter 4, by Pike et al., presents a clear critical evaluation of the practical limitations of QLS analysis for resolving the fine structure of size distributions, and describes optimal design of correlators for such purposes.

Because of the rapid increase of the light scattering amplitude of particles with their molecular weight, it is intrinsically difficult to accurately characterize small particles in the presence of larger ones. Also, the level of noise present in correlation data ultimately limits the resolution of fine structure of the relaxation spectrum. The papers presented in Sec. 2 quantitatively assess the limitations imposed on the use of QLS for particle-size determination by these factors.

Section 3 presents a large group of papers, some of which describe particle characterization by techniques related to QLS [e.g., electrophoretic light scattering and laser particle Doppler velocimetry (LPDV)], together with others which describe specific applications of QLS to problems as diverse as determining particle sizes in microemulsions to monitoring ciliary activity. I particularly enjoyed the paper by Ware et al. (Chap. 9) detailing the insights into the transport properties of charged macromolecules obtained by comparing QLS data with fluorescence photobleaching recovery experiments, the informative papers by Mazumder et al. (Chaps. 11 and 12) and I. Chabay (Chap. 13) describing particle-size measurement by LPDV, and the imaginative use of multiple detector geometries by Phillies (Chap. 10) to enable application of QLS to particle-size determination in turbid solutions. The text closes with a section comprising two interesting papers describing analysis of interparticle interactions in colloidal dispersions by QLS.

The strengths of this volume are the excellent introductory chapters, the comprehensive and critical evaluation of the utility of QLS for analysis of size distributions of colloidal particles, and the detailed presentation of QLS-based instrumentation for characterization of sols and aerosols. The book also offers a reasonably complete review of recent research activity in applying QLS to elucidate the properties of these colloidal systems. The text is inadequate, however, as a review of the extensive recent literature involving applications of QLS to investigate macromolecular transport of flexible chain molecules in solution, gel, or the bulk state.

○

\section{National Laser Users Facility Announcement}

The National Laser Users Facility (NLUF), located at the University of Rochester's Laboratory for Laser Energetics, is available for user experiments in high energy-density studies and associated applications. The U.S. Department of Energy has designated the Laboratory as a national facility, available to qualified researchers nationwide. Proposals requesting allocation of facility time should be submitted to the Manager, NLUF, by April 1, 1984. For more information about proposal guidelines and the resources available at the NLUF contact: Thomas C. Bristow, Manager, National Laser Users Facility, Laboratory for Laser Energetics, University of Rochester, 250 East River Rd., Rochester, NY 14623. 716/275-2074.

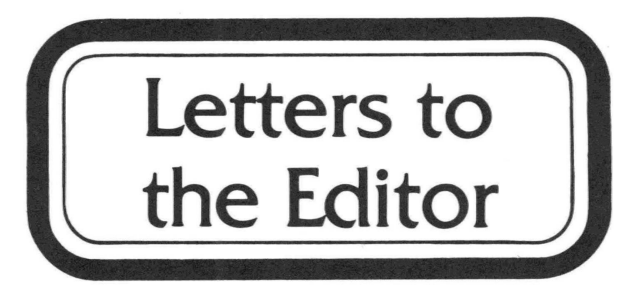

October 14, 1983

\section{Dear Sir:}

In your July/August 1983 issue, I read with intense interest your interview with Dr. Rudolf Kingslake. The following story may prove interesting to your readers.

I can remember when the military scratch and dig standards were first established they were referred to as "beauty" or "cosmetic" marks. Of course, that is exactly what they are.

During World War II in 1943, I was working at the Naval Gun Factory in Washington, D. C. I had completed their apprenticeship program in 1942 and was a journeyman precision lens, prism, and test plate maker.

One day I received a call from a friend of mine, Joe Torbert, who was a radiologist at the Norfolk Navy Yard in Portsmouth, Virginia. Joe was also a very good photographer.

Joe inquired if I could duplicate a lens for him. I replied, "I suppose so. What is the problem?" $\mathrm{He}$ stated that, as I well knew, camera equipment was impossible to obtain during this period. He said he was in a pawn shop in Norfolk, Virginia, inquiring about any cameras they might have for sale. They said they did not have any, except for an old German one with a broken lens that he could have cheap. Joe looked at the camera and saw that it was in excellent condition, except the front element of a Cooke triplet type was cracked clear through, down the middle. He bought the camera knowing that I could possibly reproduce the lens.

I asked Joe if the lens was still in its cell. He said yes, but he would take it out. I told him not to disassemble anything and asked if he had tried to use the camera. He said he had not done anything with it. I told him to take a roll of film and make some photographs of subjects of a critical nature and call me back.

A week or so later he called and said, "Bob, you won't believe this, but the photographs were of excellent quality." Joe had his own darkroom and was very careful to make top quality enlargements.

About a month or so later he called me and told me that he had won several prizes in a photo contest at Williamsburg, Virginia, with a picture he had taken with this camera. Later I saw this camera and it was cracked clear through.

The final comment I will make to this true story is, "So much for military scratch and dig standards," as Dr. Kingslake commented on in his statements.

Robert V. Harrison

Texas Instruments Incorporated

P.O. Box 226015

Dallas, TX 75266

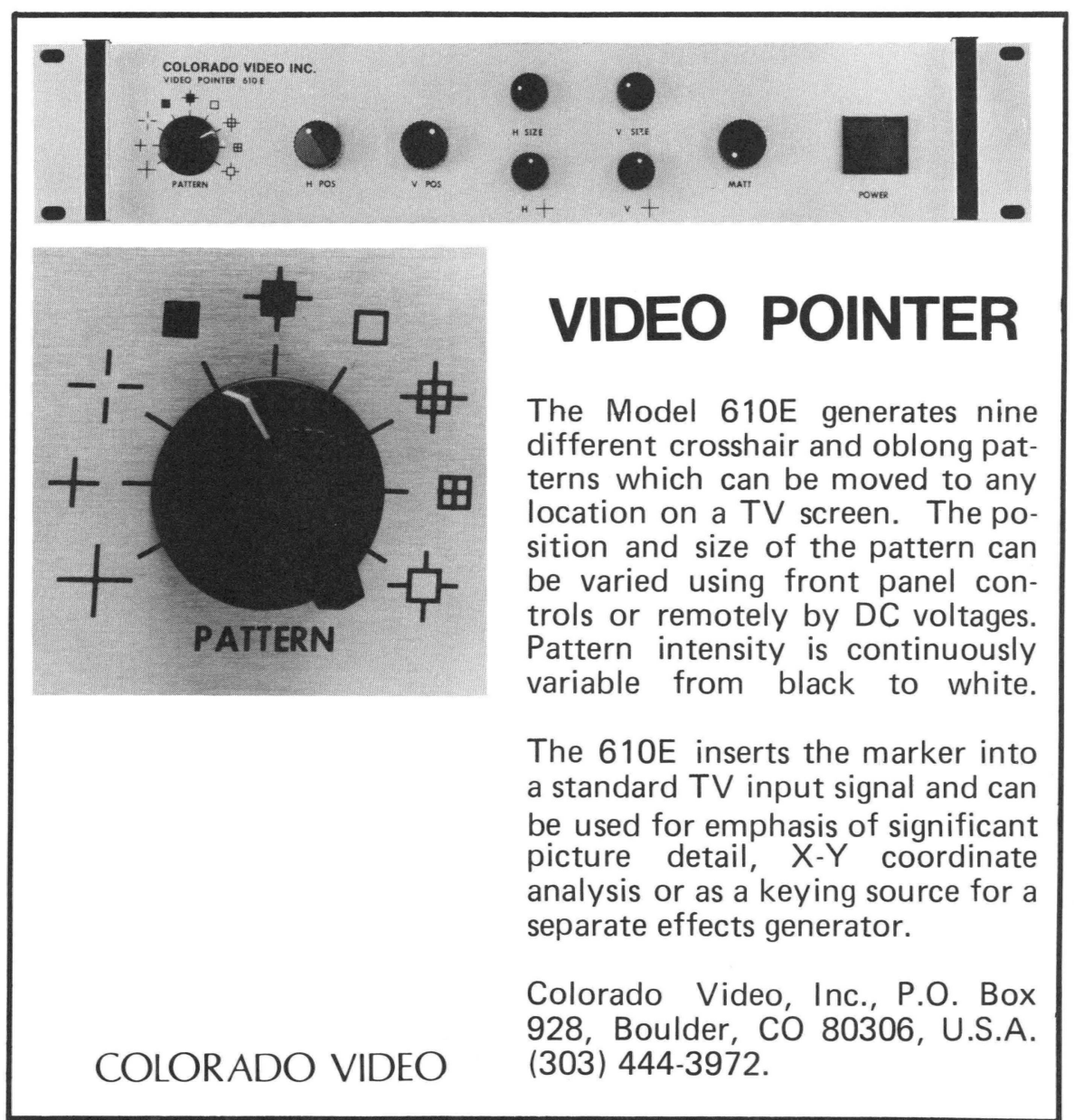

